\title{
“Nobody Other Than Me Knows What I Want": Customizing a Sports Watch
}

\author{
Piia Nurkka \\ Tampere University of Technology, Korkeakoulunkatu 1, 33101 Tampere, Finland \\ piia.nurkka@tut.fi
}

\begin{abstract}
In order for companies to effectively use customization as a design strategy, there should be understanding on what users would like to customize and why. This study explores the use of customization features of sports watch in order to assess the extent of customization, and to identify reasons for customization in this context. Survey data from 100 users of a sports watch were analyzed to understand how they use the different customization features: general preferences, functionality and appearance. The findings show that although the users vary in the use of customization, they state similar reasons for customization: control, ease-of-use, increased effectiveness, and better fit to personal preferences. The motivation to customize in this context is for the most part related to autonomy: to the sense of control the user has by having the tool to adapt the product according to own preferences, wants and needs.
\end{abstract}

Keywords: customization, sports watch, satisfaction, user experience.

\section{Introduction}

Customization features of technology are designed to provide users with control over appearance and functioning, thus increasing its personal relevance to an individual [1]. The ability to customize is a means to establish a closer connection between users and products thereby enabling them to determine appropriate product characteristics for themselves, and to meet their functional and hedonistic needs [2].

Customization as a product feature is attractive with sensible promises of benefits to customers and companies alike. Customized and more accurate offerings can provide customers with superior value [3], facilitate positive experiences, and increase satisfaction with the product [4]. Therefore, personalization may lead to positive brand associations and increase brand loyalty [1]. This can translate to increased cash flows and profitability for companies [5].

Research on customization of technological products has for the most part been confined to certain products, service contexts or points of views. For example, much research on customization of mobile and smart phones has been conducted (e.g. [6, 7, 8]). In addition, in a desk top context studies on customization can be found on PCs [9], software [10], and web site interfaces [11]. On the other hand, research has often focused on a certain type of customization, with dominance of appearance customization over functional. The different types of customization deliver different 
value for the user: appearance customization provides aesthetic value [12], whereas functional customization provides functional value improvements. Functional value, for instance as interaction efficiency [6], may lead to fulfillment of basic human needs, such as control and autonomy [11].

This study explores the use of customization features of a sports watch. The aims were to identify the extent to which customization features are used, the kind of customization features used, and the reasons for their use of customization. In addition, we wanted to understand if and how users differ in relation to customization, and if customization affects satisfaction with the product. The study gives insight into customization in a new context and the results can be used to inform design of customization that aims at enhancing user experience.

The paper is organized as follows: Section 2 starts with the definition of customization used in this research, and then reviews the related work on the topic. Section 3 describes the current study. Section 4 presents the results of the study. First, the extent of customization is reviewed generally, and secondly, by the types of customization separately. Last, the reasons for customization are reported. Finally, the results and limitations of the study are discussed in Section 5.

\section{Related Work}

\subsection{Defining Customization}

In $\mathrm{HCI}$ and related literature different terms on the concept of customization have been used. The concept means a process that changes the functionality, interface, information content, or distinctiveness of a system to increase its personal relevance to an individual [9]. However, the definition does not state who the actor in the process is: the process can be either user or system initiated. Sunikka \& Bragge [3] have reviewed the literature and suggest a personalization framework in which the user involvement in the process is at the basis and is what makes the distinction between the terms. Customization should be used if the personalization process is user (or customer) initiated, whereas personalization should be used if the system initiates the process. In user-initiated customization, the user himself manipulates aspects of his device or system or configures content of a website based on his individual needs and wants (e.g. changes the ringtone or installs a new application on a smartphone). On the contrary, in the system initiated personalization the system/device adapts content using information about the user or his behavior captured by the system/device. The personalization is implicit as the user may not be aware of it, whereas in customization the user controls the changes. Following Sunikka \& Bragge's [3] suggestion, the term customization is used in this paper. Customization is defined as user-initiated modifications to the functionality, content or interface (functionality-based customization) or appearance of the device (appearance-based customization) to increase its personal relevance to an individual. When referring to the related literature, the term customization is used instead of personalization when the question is about userinitiated process even though the term personalization may have been used in the original work. 


\subsection{Use of Customization Features}

Research suggests customization brings many positive aspects. However, to what extent do users actually use the offered customization options? Häkkilä \& Chatfield [6] examined the process of customization of mobile phones with 60 users during the first months of usage. The results illustrate active customization of the phone, with most customization occurring shortly after using the new phone for the first time. They found 11 out of the measured 17 features were customized, and nearly all had customized the most commonly customized features: the ringing tone, audio profiles and background image. The least customized features were generally more complicated to configure. Customization happened not only with fun or style features, but also with functional phone settings. A strong motivation for customization was to make the device look and feel "own" by changing the appearance of it to match the user's personal style and to reflect his interaction preferences. Interestingly, Ventä et al [7] report a contrary finding regarding the appearance customization of a mobile phone. In their interview study of 40 mobile phone users, the users stated that they bought a phone with looks they liked, and thus did not want to modify it afterwards.

Furthermore, Tossell et al. [8] studied the customization of a smartphone with 24 participants during the first two months of usage. The aim was to examine the relationship between personalization, device usage and usability. The results show a high range of customization, but not across every customization feature measured. For instance, every user acquired a case for their smart phone, but the number of applications installed differed. In general, the results show there are differences between users in the use of customization: not all users customize their smartphones and females and males customize their smartphones differently. A relation between customization extent and usability was found: those who customized their phones more tended to rate it as more usable. In addition, the users who customized more also used their device for greater periods of time on a broader range of applications.

In a study by Page et al [10] the typical customizations made on a word processor were identified. Most users had made changes to their general preferences settings when only few customized the visual appearance of the interface. However, almost all, ninety-two percent of the users, had customized their software in some way. Most of the customization was done to facilitate the participants' work practices, and consequently, users who used the software most did the most customization.

To sum up, users vary a lot on how many features they customize. Features relating to appearance customization are often among the most customized. However, customization is also performed to increase ease of use. Consequently, high level of customization may increase the time of use of the product.

\subsection{Reasons and Motivations to Customize}

Customization, by letting users modify their products and services, assumes users are active actors rather than passive consumers. The question why to customize has therefore attracted researchers. For example, Oulasvirta \& Blom [1] applied modern theories of motivation to explain user behavior. According to them, analyzing motivations 
is necessary because they determine the personal relevance that an act of customization carries. However, users differ in how strongly they express different motivations for customization. Oulasvirta \& Blom [1] claim there is no special need for customization; rather there are context-independent basic needs that are idiosyncratically manifested motivations related to the use of a product's features. Specifically, users are willing to customize when the product involves and nurtures their psychological needs of autonomy, competence, and relatedness, taps into and extends their interests and preferences, and makes it possible for a user to transform a uniform technology to personally useful and enjoyable tool that can be used to improve and enjoy life and work. Thus, even though there is no need as such for customization, appropriate design of customization features is crucial: "It is through customization features that technology can help the user to align her inner motivational resources with her actions." [1, p.13].

In addition, Marathe \& Sundar's [11] study on customization of a web portal reveals that customization has deep psychological value when users take time to engage in it and make changes according to their preferences. Specifically, their data show that customization is associated with two psychologically meaningful gratifications: sense of identity and sense of control. Their results demonstrate how customization leads to two distinct patterns of effects: while the effect of customization on sense of control was fully mediated by sense of identity, its effects on sense of identity was only partially mediated by sense of control. This finding leads to the conclusion that "having control is all about being able to express one's identity, but feeling a sense of identity has implications beyond feeling sense of control" [11, p.787]. From an interface design perspective, customization features should therefore offer possibilities for self-representation to express identity. UI designers need to consider what gratification each customization feature will imbue in the user: by fulfilling higher-order needs like enhancing identity and control, designer can create an interface that is an end in itself rather than a means.

\subsection{Dispositions to Customize and Effects of Customization}

The theory of personalization of appearance (TPA), developed by Blom \& Monk [3] and elaborated by Monk \& Blom [13], indicates the dispositions to personalize and the following effects of personalization on user. The theory has been derived from studies on the personalization of web portals, mobile phones and computer desktops. The dispositions are categorized as the user-, system- or context-dependent and each of them has individual dispositions. For example, user-dependent dispositions are: the frequency of use of the system, ownership of the system, knowledge of personalization and usage time. The system-dependent dispositions relate to the ease and cost of personalization and to the effectiveness of personalization items, i.e. their ability to elicit emotions or improve ease of us. Presence of a disposition may increase the personalization behavior, however, the authors do not explain how many or which of the dispositions are crucial for the personalization to happen. Nevertheless, personalization brings about socio-emotional effects on user. Authors have identified effects that can be divided into Cognitive, Enduring Emotional, Transient Emotional and Control Effects. 


\section{Study on Customizing a Sports Watch}

An online questionnaire was designed to provide information on the extent and the reasons to customize a sports watch (Figure 1.). In this section, first, the product and measured customization is explained, followed by the description of the online questionnaire, respondents and analysis of the data.

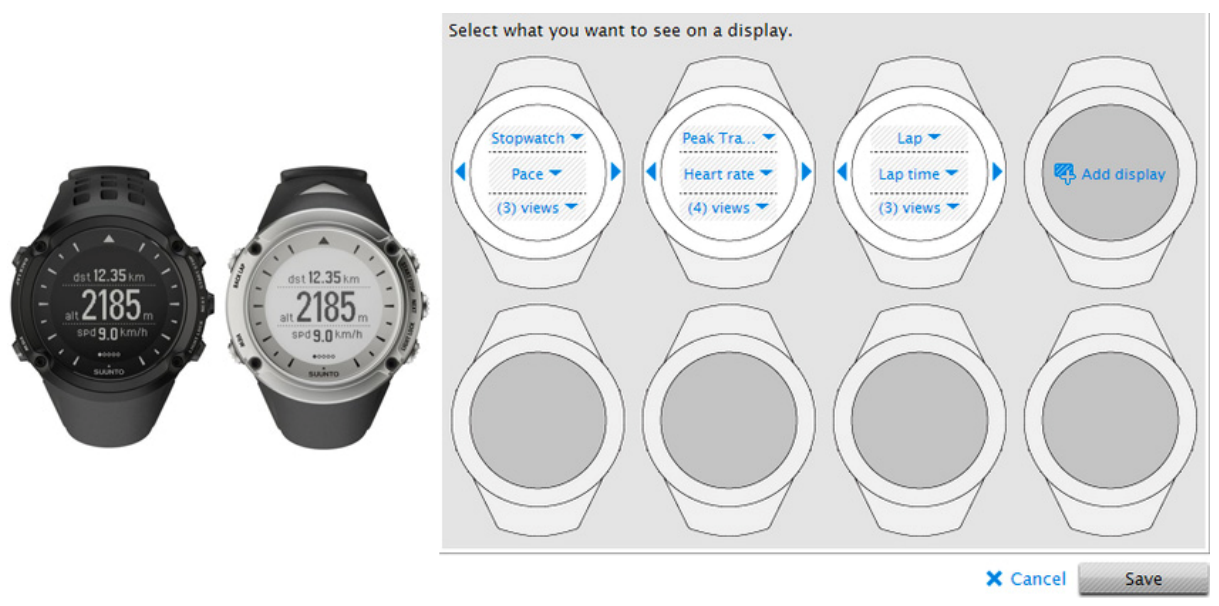

Fig. 1. One the left, the two different variants of the sports watch: the black with dark display and the silver with light display. On the right, a screen capture of the customization of running mode on the online training log

\subsection{Customizing the Sports Watch}

The product under investigation is a sports watch called Ambit produced by Suunto a Finnish developer and manufacturer of dive computers, heart rate monitors and outdoor sports instruments. The watch has an integrated GPS and the possibility to monitor heart rate. In addition, it has diverse set of specialized outdoor and training functions and is intended for (according to the marketing of the product) "the Outdoor Explorer" [14]. The product is a part of a cross-platform system in which the other part is an online training log site. The site can be used to store and manage exercise logs and to customize the device.

To form an understanding of the extent of customization with this type of device we first identified all the features or items that could be customized on the device. Altogether 28 different features were identified as customizable. However, some features (for example the seven items related to personal settings, e.g. body weight) might not be considered as customization items as such, but rather as essential features to adjust according to the user's characteristics in order for the product to work as intended. Therefore we used the following criteria in the selection of items to be included in the study: the items should concentrate on the top-level customization that are central to the product use, the items should represent the main activities of customization based on previous research $[8,10]$ and at least some of the items should be 
customizable both on the device and on the online training log site. Due to the selfreport method, it was important that it was reasonable to assume the users were aware of the customization options that were included in the questionnaire. Consequently, we concentrated on features that are explained in the user guide of the product in chapters 'Customizing Your Product' and 'Adjusting Your Settings'. Based on these criteria, ten different measures of customization in three different categories (functionality, general preferences and appearance) were chosen to be included. Top-level customization in this study refers literally to the highest level of customization, namely to the degree whether customization was committed or not on the chosen categories and their main sub-categories. For example, the extent of customization within a default exercise mode (e.g. how many displays and which data fields were customized) is out of the scope of this study.

Customizing the Functionality. Customizing the functionality of the device can only be done on the online training log site. The customization of the functionality of the device happens on two levels: first by choosing what exercise modes are stored on the device and second, by determining what is being measured and shown on the screen during the particular exercise. Consequently, this type of customization defines (part of) the content, and affects the interface of the device when using it during exercising. For example, less exercises stored in the device improves getting access to the functionality, and less screens attached to an exercises decrease the need for scrolling and therefore improves usability.

As a default there are eight different exercise modes stored in the device: alpine skiing, cycling, indoor training, mountaineering, running, trail running, trekking, and other sports (see Figure 2 for a screenshot of customizing the mode for running). The default modes can be used as they are, or they can be customized in the online training $\log$, after which they need to be downloaded on to the device again. In addition, new modes for new exercises can be created from scratch and later modified if needed. In order to measure the top-level customization of functionality we asked if the user had:

- Edited displays to default exercise modes

- Added displays to default exercise modes

- Deleted default exercise modes

- Created new exercise modes

- Edited new exercise modes

Customizing General Preferences. Customizing general preferences was included because this type of customization can be performed both on the device and the online training log site. In addition, customizing general preferences has been found to be one of the main customization activities [10]. Of the options for general preferences, customizing formats of time, date and units was included in this study. Customizing the time and the date affects how the display looks, and customizing the unit system affects how the data is displayed while exercising. As a default, the device had as a time format $24 \mathrm{~h}$, as a date format dd.mm.yy and the unit system was set to metric. Thus, if the user was satisfied with these formats, no customizing was needed. In the study, the user was asked if he had: 
- Customized time format (12h or $24 \mathrm{~h})$

- Customized date format (dd.mm.yy or $\mathrm{mm} / \mathrm{dd} / \mathrm{yy}$ )

- Customized unit system (metric, imperial or advanced)

Customizing the Appearance. According to previous research customizing the appearance is an important type of customization and is deeply rooted in the use of certain products [13]. However, with the device, there are limited possibilities for customization: the display can be inverted from dark to light, and vice versa (both on the device and on the online training log) and the strap can be changed. Customization of the appearance of the device was measure with the two measures:

- Invert the display

- Change of strap

\subsection{Customization Questionnaire}

The study was carried out as a part of larger research project investigating long term user experience during six months of product usage. During the research project, each month the respondents answered a questionnaire in English on their product usage and experiences with the product. The customization questionnaire reported in this paper was included in the third survey (third month into the study). Some basic demographic data (e.g. age and nationality) and data on sports activities collected at the recruitment phase of the study are included here. In addition, data on exercise habits, product usage and satisfaction with the product collected in each survey are combined with the customization data.

The 21 questions related to customization consisted of both closed and open-ended question, and were on the last page of the questionnaire. At top of the page, a description of customization was given. Multiple choice questions were used to find out about the extent of customization: the respondents were asked to mark from the list of ten customization items all the ones they had used during the total time of product usage. Closed-ended questions with a 7-point interval scaling (1-7; 7 signifying strong agreement) were used to evaluate the importance of customization, the ease of customization and the effects of customization (e.g. product being more useful and personal after customization). Open-ended questions were used to probe about the knowledge of customization of the product before taking it into use and the reasons for customizing the product. In addition, the respondents were encouraged to comment freely after questions.

\subsection{Respondents}

The respondents for the six month long study were selected among the customers who responded positively to the e-mail invitation to take part in the study by answering a short questionnaire with basic demographic, product purchase and usage questions. The invitation was sent to 521 registered owners of the product of which $190(36 \%)$ expressed their interest to take part. 121 were chosen to take part based on three criteria: 1 . Short usage time of the product; 2. Nationality; and 3. First come first served. As an incentive to take part, a respondent would receive a product of Suunto worth up to 269 
USD after completing all six surveys.At the time of the data collection for this paper (third survey), the number of respondents had dropped to 110 (21\% of the whole sample getting an invitation). A further ten respondents and their responses were removed from the analysis due to missing data leaving the sample size to 100 respondents.

\subsection{Analysis}

For the quantitative data, nonparametric tests were selected due to the nonparametric (distribution-free) nature of the gathered data. The CHI Square was used in pairwise comparisons of categorical variables and Spearman's $\rho$ was used in calculating correlations. A content analysis was performed to analyze the participants' descriptions of their main reasons for customizing the device. In the analysis, the basic needs of autonomy, competence and relatedness, and the motivations relating to them were used as the guiding framework [1].

\section{$4 \quad$ Results}

\subsection{Characterizing the User}

Demographic Information. The 100 respondents consisted of 93 males and 7 females, and their age varied between 22 and 64 years, with the mean age being 41 years (two modes: 37 and 45 years). 49 respondents had owned the device for three months and 51 respondents for four months. Over half of the respondents were from Europe (58 respondents), but the sample consisted nationalities from almost every continent: North America 18, Oceania 11, Asia 10, and (South) Africa 3. There were 17 different nationalities among the respondents. The four biggest nationality groups were Dutch with 13 respondents, Americans with 12 respondents, Finnish with 10 respondents and Australians with 9 respondents. 34 respondents have English as their mother tongue.

Sport Activities. Since the product is targeted for training and especially outdoors activities, we were interested in what activities the respondents engage in. From the list of 18 types of sports (with an open text for 'other' option), the respondent marked each sport he does. The respondents are active: on average they engage in seven different sports (range $2-16$, mode 6), and altogether 46 different sports were mentioned. The most often mentioned sports were hiking (81), road running (70), trail running (60), road bicycling (60), going to the gym (49) and mountain biking (42). Thefavorite sport was asked with an open-ended question. Running was the favorite sport with 47 replies (includes 27 replies mentioning trail running specifically). Other top three sport activities were hiking and biking (off road, mountain or road) both with 14 mentions and skiing (off-piste, alpine or Nordic pole) with 7 mentions. On average, the participants exercise 4.3 times per week (STD 2.08, median 4, mode 3,).

Sports Watch Usage. The respondents were asked how often they use the device as a daily watch and in exercising (Table 1). Although the device is mainly targeted for use during different exercising activities, over half of the respondents (56) use it daily as a daily watch. 
Table 1. Frequencies of the respondets using the watch in exercising and as a daily watch

\begin{tabular}{|c|c|c|c|c|c|c|}
\hline & & \multicolumn{4}{|c|}{ Using the watch in exercising } & \multirow[b]{2}{*}{ Total } \\
\hline & & $\begin{array}{c}\text { Less than once } \\
\text { week or not at } \\
\text { all }\end{array}$ & $\begin{array}{c}\text { Once a } \\
\text { week }\end{array}$ & $\begin{array}{l}\text { Several times } \\
\text { a week }\end{array}$ & Daily & \\
\hline \multirow{6}{*}{$\begin{array}{l}\text { Using the } \\
\text { watch as a } \\
\text { daily } \\
\text { watch }\end{array}$} & Not at all & & 2 & 3 & & 5 \\
\hline & $\begin{array}{l}\text { Less than once } \\
\text { week or not at all }\end{array}$ & 1 & & 7 & 1 & 9 \\
\hline & Once a week & & 1 & 10 & & 11 \\
\hline & $\begin{array}{l}\text { Several times a } \\
\text { week }\end{array}$ & & 1 & 13 & 5 & 19 \\
\hline & Daily & 2 & 5 & 33 & 16 & 56 \\
\hline & Total & 3 & 9 & 66 & 22 & 100 \\
\hline
\end{tabular}

However, diverse use of the device is common: majority of the respondent (67 respondents) falls under a usage profile in which the watch is used at least several times a week both in exercising and as a daily watch. 16 participants use the device daily both as a time piece and in exercising. On the other hand, there are respondents who tend to use the watch more specifically for exercising: 21 respondents, who use the watch several times a week in exercising, use it only up to once a week as a daily watch. In addition, there are 8 respondents who mainly use the watch as a daily watch and only up to once a week in exercising. Furthermore, 4 respondents use the watch rarely in either use on a weekly basis.

\subsection{The Extent of Customization}

Table 2 gives the percentages of the 100 respondents that reported having customized that particular item. The most popular item to be customized was to invert the display. Almost as popular was the functionality related customization of editing displays to default exercise modes. However, overall, the rate of customizing was high with every item with the exception of customizing the appearance by changing the strap which was done by only one respondent.

Table 2. The percentage of the respondents who customized the particular item

\begin{tabular}{|l|l|c|}
\hline Customization type & Item & Percentage \\
\hline Appearance & Invert the display & $83 \%$ \\
\hline Functionality & Edit displays to default exercise modes & $82 \%$ \\
\hline Functionality & Add displays to default exercise modes & $74 \%$ \\
\hline Functionality & Create new exercise modes & $68 \%$ \\
\hline Functionality & Delete default exercise modes & $58 \%$ \\
\hline General preferences & Customizing Time format (12h or $24 \mathrm{~h}$ ) & $56 \%$ \\
\hline General preferences & Customizing Unit system (metric, imperial or advanced) & $51 \%$ \\
\hline Functionality & Edit new exercise modes & $48 \%$ \\
\hline General preferences & Customizing Date format (dd.mm.yy or mm/dd/yy) & $43 \%$ \\
\hline Appearance & Change of strap & $1 \%$ \\
\hline
\end{tabular}


Customization Score. The customization score represents the number of the customization features the respondents reported having used. On average, the respondents had used 5.6 (STD 2.2, median 6, mode 7) of the ten customization features measured in this study. The high mean score indicates relatively comprehensive use of the features available, and may suggest general interest to customize in this context. However, the wide range of customization (range $0-9$ items) and high variability across items measured among the respondents suggests there are differences in customization behavior. Grouping the respondent based on the number of items customized show there are four main groups (Table 3) that can be divided to low customization group and high customization group with the average customization score as a divider.

Table 3. Groups of respondents based on the number of items customized

\begin{tabular}{|c|c|c|}
\hline Customization group & Number of items customized & Frequency \\
\hline \multirow{2}{*}{ Low customization } & $0-3$ & 19 \\
\cline { 2 - 3 } & $4-5$ & 27 \\
\hline \multirow{2}{*}{ High customization } & $6-7$ & 34 \\
\cline { 2 - 3 } & $8-9$ & 20 \\
\hline
\end{tabular}

Earlier studies have found differences in customization extent, for example, originating from gender [8] and the presence of dispositions [9]. The effect of gender was impossible to measure in this study due to small amount of females in the sample. However, user related dispositions 'Frequency of Use of Product' and 'Knowledge of Customization', and system related disposition 'Ease of Customization' were taken into account in this study. A Chi Square test was performed to determine if there was a difference between usage frequency of the device in exercising and use as a daily watch and customization score (low customization/high customization group). The test indicated a significant difference with usage in exercising $(\chi 2=7.19$, df $=2, p$ $<.05)$. However, the test failed to indicate a significant difference with usage as a daily watch $\left(\chi^{2}=2.74, \mathrm{df}=3, \mathrm{p}=.433\right)$. The results indicate that use of the device in exercising may urge customization. Furthermore, a Chi square test was performed to determine if there was a difference between knowledge of customization and customization score. The respondents were categorized based on their knowledge of customization in three groups: 'Being able to customize was one of the purchase decisions', 'I knew about customization before purchase' and 'I did not know about customization before purchase'. However, the test failed to indicate a significant difference $(\chi 2=2.72$, df $=4, p=0.605)$. Correlation (Spearman's $\rho$ ) was calculated to study the relationships between the ease of customizing the functionality and the customization score. There was a weak correlation between customizing the functionality and customization score (Spearman's $\rho \mathrm{r}=.209$, $\mathrm{p}<.05$ ). This correlation indicates that there is a relation between the ease of customization of exercise modes and customization behavior, but it is impossible to say if ease of customization caused more customization. It may as well be the opposite: by customizing more the respondents may have enhanced skills in customizing and therefore it is perceived as easy. 
In the following paragraphs the use of each of the different types of customization will be discussed separately.

Functionality-Based Customization. Customization of functionality was measured by three items relating default exercise modes (editing displays, adding displays and deleting modes), and two items relating to new modes (creating and editing).

The possibility to customize the default exercise modes was used extensively: 87 respondents had utilized at least one of the customization options provided. 50 respondents had used all the three options given, 21 had edited and added displays, 6 had only edited displays, 5 had edited displays and deleted modes, 2 had deleted modes, 2 had only added displays and one had only added displays and deleted modes. 68 respondents reported having used the customization option to create a new exercise mode, and 48 had also edited the new mode. 38 respondents had customized all three default modes and created new modes. It was more popular to customize the default exercise modes than to create their own. A Chi Square test was performed to determine if there was a difference between the usage frequency of the device in exercising and the use of functionality customization items. The test with create new mode indicated a significant difference $(\chi 2(2)=6.16, \mathrm{p}<.05)$ suggesting that the respondent who use the device more in exercising create more new exercise modes.

General Preferences. Formats were also actively customized: altogether 71 respondents had customized at least one of the formats. To be precise, 35 respondents had customized all three formats; 27 respondents had customized just one of the formats (time by 13 respondents, unit by 11 and date by three), and nine respondents had customized combination of two formats. Altogether eight different combinations to customize (or not to customize) the three formats were found.

There are different conventional ways to present formats in different countries and we expected respondents from countries where the default formats are commonly used not to customize them. A cultural analysis was performed due to the unexpected high number of respondents who customized the formats. Among the 19 different nationalities there were 10 from countries (origins of 51 respondents) in where the same as the default date, time and unit formats set on the device are commonly used. In eight cultures (origins of 37 respondents) partly same as default formats are used: for example in Australia the dd.mm.yy style of date format and metric system is used, but for the time, the $12 \mathrm{~h}$ format is used. In some countries, like Canada and Philippines (origins of 7 respondents), different notations, especially with regard to date and time, are used concurrently depending on the language of the citizen. In contrast, in US (12 respondents) none of the default formats are used suggesting a need for customization of formats for American respondents in our study.

Based on the clarification of the conventions in different countries, we investigated the customization of each format separately per respondent by comparing the respondent's nationality to the reported customization of the formats. The base line was the assumption that a respondent would use formats customary to his culture. This analysis aimed at investigating the possible effect of culture on the reported customization of formats by revealing frequencies of respondents who 1) customized according to expectations based on cultural notation and 2) customized contrary to cultural 
notation. Some respondents were left out from the analysis due to uncertainty of commonly used notations in their countries of origin: British and Canadian respondents (14) were left out from the analysis of time, Canadian, Filipino, and South African respondents (10) were left out from the analysis of date, and British were left out from the analysis of units.

Table 4. Customization behavior based on cultural notations

\begin{tabular}{|c|c|c|c|}
\hline & Date $(\mathbf{n}=\mathbf{9 0})$ & Time $(\mathbf{n}=\mathbf{8 6})$ & Units $(\mathbf{n = 9 2 ) :}$ \\
\hline According to expectations & $62 \%$, & $65 \%$, & $55 \%$, \\
\hline Against expectations & $38 \%$ & $35 \%$ & $46 \%$ \\
\hline
\end{tabular}

The results indicate individual preferences differ from cultural conventions with approximately third of the respondents. The difference from cultural conventions is further highlighted when investigating the customization of the formats with the biggest nationality groups further. For example, five out of twelve of the Dutch respondents had done some customization: one had customized the date and time format, one the date and unit, two had customized the unit and one the time. Further, five out of ten of the Finnish respondents had customized all the formats, one had changed the time and one the date. This leaves only three who had kept the default formats that are the common notation in Finland. In addition, half of the American respondents (6/12) had customized all formats, two the date and time, one time and date, one time and unit, one the date, and one had not customized any.

To understand the reason for customizing the general preferences contrary to cultural conventions, we looked into the responses to the open-ended question asking reasons for customizing the device (Analyzed more thoroughly in section 4.5). Since the question was general, only nine respondents specifically mentioned reasons to customizing the formats. Nevertheless, the responses show that convenience in a context or in use situation and willingness to experience and try out new things may trigger the customization of formats:

"I change the unit system for pacing a friend during a 100miler in the USA. I used the imperial system instead of the metric so we didn't have to do the conversion all the times" (ID64, Canadian)";

"I liked trying different formats and using US formats vs European (...)" (ID43, American).

Customization of Appearance. There were only two possibilities to customize the appearance of the device: by changing the strap and by inverting the display from light to dark or vice versa. The latter was the most often customized item.

Only one respondent had changed the strap. Analysis of the free text comments showed that many respondents were not aware of the possibility to change the strap but would be interested in it. On the other hand, there was couple of respondents who were not interested in customizing the appearance at all:

"No need to customize look. This one is perfect." (ID91). 


\subsection{Reasons to Customize}

In the analysis of qualitative data on reasons to customize, two responses were excluded due to answers relating more to the need for new customized features than reasons to customize. The analysis was based on Oulasvirta \& Blom [1] who suggest the Self-Determination Theory (STD) [15] as a framework to understand customization. The reasons were categorized into the motivations relating to the attainment of three basic needs, autonomy, competence and relatedness.

Four respondents claimed they have not engaged in customization (yet) or that they are happy with their settings. These four respondents were on the low customization group (0-3 items customized). The responses from the remaining 94 respondents gave 157 reasons to customize: each reason given was categorized and thus, the average amount of reasons per respondents was 1.6. The results are presented below first with Figure 3 presenting frequencies of the reasons as motivation categories. After that the main findings on each motivation is described separately with quotes from the respondents. A category "Looks" was included in the analysis outside the analysis framework since responses relating to the improving the appearance did not fit to any of the categories.

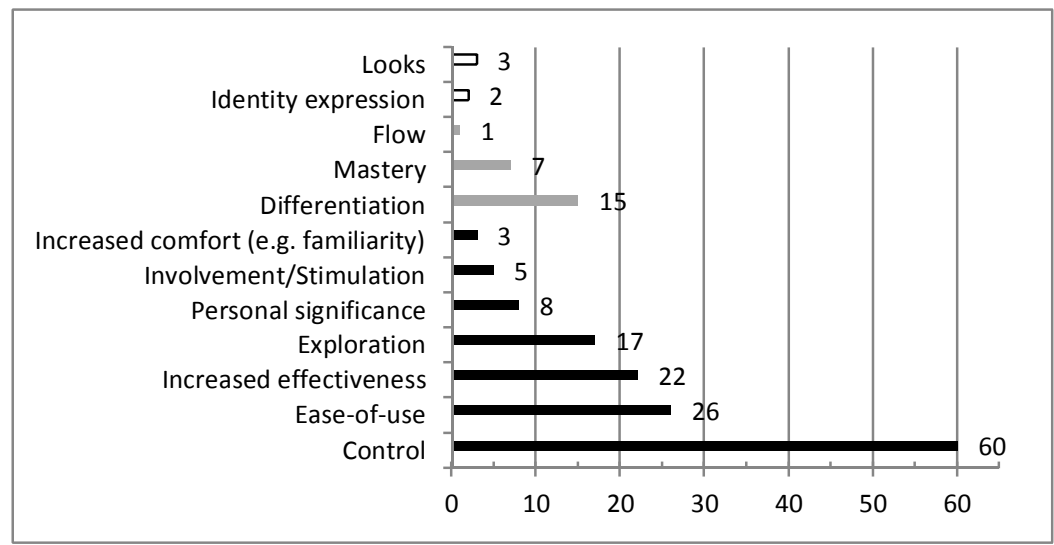

Fig. 2. Reasons to customize categorized in motivation types relating to autonomy (black), competence (grey) and relatedness (white). The graph presents number of reasons given by the respondents for each motivation type

Autonomy. The given reasons to customize were clearly most often related to control and the motivation relating to autonomy. This was highlighted by the use of wordings like "I want", "I need", "I like to have". The feeling of control was often related to ease-of-use and/or effectiveness of use. In addition, respondents often described the specific needs in relation to their activities: the importance to see the relevant information easily in a glance and without the hassle of going through different screens. At the same time, they recognized customization as "mandatory" in order to make the device personal. Some respondents engaged in customization just for fun or to try out. 
"No company could make a watch which suits everybody, so customizing is mandatory for me. Nobody other than me knows what I want." (ID21)

"The main reason is that I want to decide what information is important to me while I exercise. I also want to control what buttons and how often I have to push a button in order to get the information I need." (ID54)

"Just to try out how it works, to have kayaking in the menu, and for having specific details on the watch display for different exercises to make it easier to use." (ID36)

Competence. Motivations to customize relating to the basic need of competence are to do with acquiring skills, differentiating oneself by preferences and interests and engaging in action. Often the competence motivations were mentioned along with control and the benefits of customization was acknowledged. In some responses the ability to customize or to learn from the act of customization was seen as beneficial to the individual.

"I deleted different sport profiles that I knew I would never, or rarely use. The same goes for the custom screen displays - I only display the parameters that I know will benefit me during training." (ID90)

"Also, I have a better understanding of the functions available and have been to fit the best functions to certain exercise types." (ID107)

Relatedness and the Looks. Customization of the device was clearly not driven by motivations of emotional or identity expression. There were many who claimed the reason for customization was to make the information provided by the device more personal but only two respondents claimed they customized the device (and not only the information) to make it their own. Three respondents specifically said they had changed the display color to improve the looks although most respondents had used the feature.

"I have changed to the dark display because it looks better. I have customized the exercise mode to also allow me to measure other activities and display I like to have while I am performing said exercise. I set the time format to military time (24 hrs) which is my preferred way to tell time." (ID77)

"Needed to customize the watch to make it feel more personal, to make it feel like it belongs to me. To make the intepretation of information from the watch easier." (ID106)

Summary. Both the low and high customization groups expressed for the most part similar reasons to customize. The main differences with them were that in the high customization group's responses control appeared more, whereas the low customization group mentioned motivations relating exploration more often.

\subsection{Importance and Effects of Customization}

Importance of customization and effects of customization (customization makes the product more personal and useful) was measured by asking an agreement to corresponding statements with a 7-point scale (1-7; 7 signifying strong agreement). In addition, the respondents had possibility leave comments on a free text field. Correlations 
(Spearman's $\rho$ ) were calculated to study the relationships between the statements and customization score. Table 6 presents the basic descriptives and correlations with customization score. Generally, the respondents showed high agreement with all statements. Regarding the importance of customization, the results suggest it is more important for the respondents to customize the exercise modes than the basic functionality or appearance. In addition, the ability of customization to make the product more useful was appreciated:

"I like to customize my gadgets for my preference. It's like new every time I customize it." (ID101)

There was a positive correlation between all statements and customization score. This correlation indicates that respondents who expressed stronger agreement with the statements had customized the product more.

Table 5. Basic descriptives and correlations of customization score with statements relating to effects of customization

\begin{tabular}{|l|c|c|}
\hline Statement & Descriptives & Cust. score \\
\hline $\begin{array}{l}\text { Being able to customize the exercise modes is } \\
\text { important to me. }\end{array}$ & 6.7 (STD.63, median 7, mode 7) & $.340^{* *}$ \\
\hline $\begin{array}{l}\text { Being able to customize the formats of time, } \\
\text { date and units is important to me. }\end{array}$ & 5.9 (STD1.8, median 6, mode 6) & $.328^{* *}$ \\
\hline $\begin{array}{l}\text { Being able to customize the looks of the product } \\
\text { is important to me. }\end{array}$ & 5.1 (STD1.7, median 5, mode 7) & $.228^{*}$ \\
\hline $\begin{array}{l}\text { Being able to customize makes the product } \\
\text { more useful. }\end{array}$ & 6.7 (STD.68, median 7, mode 7) & $.337^{* *}$ \\
\hline $\begin{array}{l}\text { I feel the product is more personal after custo- } \\
\text { mization. }\end{array}$ & 6.5 (STD.80, median 7, mode 7) & $.347^{* *}$ \\
\hline$* *$. Correlation is significant at the 0.01 level (2-tailed), *. Correlation is significant at the 0.05 level (2-tailed). \\
\hline
\end{tabular}

\subsection{Satisfaction}

Satisfaction with the device was measured with a simple question: "How satisfied are you with your Suunto Ambit?" with a 7 -point scale (1-7; 7 signifying strong satisfaction). The respondents reported very high satisfaction with an average score of 5.97 (STD 1.2, median 6, mode 6). Correlation (Spearman's $\rho$ ) was calculated to study the relationships between satisfaction and customization score but no correlation between the measures was found (Spearman's $\rho$ r=-.013).

\section{Discussion}

The current results show the users of a sports watch customize their device extensively albeit a high customization group and a low customization group could be distinguished. There was only one respondent who had not customized his device at all and only one customization option (appearance-related: strap change) was used by only one respondent. However, little use seemed to be due to unawareness of the respondents about the option, and not because it was not appreciated. Customization of 
appearance (invert the display) was the most often customized item followed by the four main items of functionality customization. Functionality-based customization was rated as the most important, and customization was seen as an instrument that makes the product more useful. The respondents seemed to have practical reasons for customization: those who used the device more in exercising had also customized it more extensively. However, we cannot say if more customization resulted in more use (in exercising), a phenomenon seen with smartphones [8], rather, the product may be contrasted to a work tool: in Page et al [10] study on a word processor persons who used the software most, had customized most. This was also the case here: the respondents who used the device more in exercising, had customized it more. However, the finding does not allow proposing a cause-and-effect relationship between the variables. Rather, it may point to the fact that using the device more in exercising, the user becomes more aware of his needs with regard to measuring his performance and capabilities of operating the device during the particular exercise (e.g. contextual factors). Therefore, more needs for customization arouses, and more customization on the device is likely to be performed. The sport a user does may have an effect on customization needs.

In previous research the emphasis has often been on the customization of the appearance (e.g $[9,13])$ because customization of appearance is the way to make the device look and feel "own" [6]. The results of this study suggest that customization of appearance is not the only condition to feel that a product is personal. Being able to customize the main functionality of the products, to better fit it to the individual needs (in an activity important for the individual), can bring about the feeling of the product being personal. In fact, the findings by Marathe \& Sundar [11] on the effect of customization on sense of control seem to fit here: having control is a way to express one's identity. Furthermore, it the results indicate that the respondents got a product with looks they liked and thus, customization of appearance was not so important at least in order to express identity.

Control was the main motivation to customize with other motivations relating to autonomy following. No major differences between the respondents in different customization groups were found, but high customizers expressed some more control, whereas low customizers expressed more motivations relating to exploration. Overall, the results suggest that customization can be explained by the three basic needs, autonomy, competence and relatedness, with motivations in this context deriving mostly from the need for autonomy. Customization features are valued due to the sense of control the user has by having the tool to adapt the product according to own preferences, wants and needs. This sense of control was seen as a reason to customize in every customization type, not only in functionality customization.

The respondents who did not customize or customized minimally raise the question on their reasons for not customizing. In line with the results from Tossell et al. [8], it was learned that non-customizers have reasons for their behavior: there was not a need for it, the options were not suitable or the user was not aware of the possibilities. We feel the reasons for non-customizing may point to directions to further develop the customization features or, at least, improve the information about them. 
Finally, we did not find correlation between satisfaction and customization extent despite it seems logical. A reason for this may be the overall high satisfaction with the product, and the insight that a user does not need to customize extensively to be satisfied with a product as long as his personal needs will be fulfilled and he can feel to be in control. Furthermore, a small matter, like the inversion of display color may be enough for a pleasurable experience.

Design Implications. Customization is a product feature that enables differentiation from competitors. However, available options should be easily found and recognized by the users or they may be unused despite their interest in using them. It seems there cannot be too many options: the more the better. The user should have the full control in deciding what is customized, but recommendations, for example, relating to contextual factors might be in order.

Limitations and Future Studies. There are certain limitations with this study. First, as the data was collected with an online questionnaire we cannot be sure how seriously the respondents took their reporting task, and since no observation was possible, the results are solely based on self-reports. In addition, it is not known if the respondents fully understood the concept of customization. On the other hand, the method seems appropriate for studying the top-level customization, and respondents with missing data were excluded from the analysis. Another limitation relates to the respondents that were drawn from the company's customer base. A customer interacting with a company might avoid giving negative feedback especially in a study with an incentive. However, the respondents were informed that the study is a research project conducted in a university and for example, all the communication with the respondents was from the university. In addition, reaching the respondents would have been quite difficult without the help from the company. Finally, the study reviewed the toplevel customization of a sports watch: if more features were included to be measured, there might be more variety in the customization profiles.

In future studies, the aim will be to explore the true effect of customization on satisfaction and user experience by collecting data with repeated measures from the first usage (before any customization is performed) into couple of months use. With repeated measures the change in satisfaction and user experience could be compared to the extent of customization. At the same time, the process of customization could be explored: how often is customization performed and why, are the customized features actually used, and what kinds of effects arise from the process. Similarly, the preferred ways to do customization could be investigated. Another point of direction could be to study customization of a couple of products from the same user to explore the customization behavior and gain understanding in customization and motivation to customize in different context.

\section{Conclusions}

This study gives insight into customization in a new context. Previous studies have for the most part concentrated on the customization of appearance; this study 
introduces functional customization as an important part of product experience. This study explores the use of customization features of sports watch in order to assess the extent of customization, and identify reasons for customization in this context. Survey data from 100 users of a sports watch were analyzed to understand how they use the different customization features: general preferences, functionality and appearance. The findings show that in this context customization was extensive, but a high and a low customization groups were found. Despite the differences in customization extent, the users state similar reasons for customization: control, ease-of-use, increased effectiveness, and better fit to personal preferences. The motivation to customize in this context is for the most part related to autonomy: to the sense of control the user has by having the tool to adapt the product according to own preferences, wants and needs.

Acknowledgments. The author thanks Suunto and especially Tiina Taskinen for cooperation and enabling of the study, researcher Tiina Koponen (Human-Centered Technology, Tampere University of Technology) for her invaluable contributions to help carrying out this study and assistant professors Timo Partala and Satu JumiskoPyykkö (Human-Centered Technology, Tampere University of Technology), for their comments on the manuscript. This research was supported by the Finnish Doctoral Program in User-Centered Information Technology (UCIT) and the Finnish Funding Agency for Technology and Innovation (Tekes) through the project DELUX.

\section{References}

1. Oulasvirta, A., Blom, J.O.: Motivations in personalisation behaviour. Interacting with Computers 20, 1-16 (2007)

2. Weightman, D., McDonagh, D.: People are doing it for themselves. In: Proc. DPPI 2003, pp. 34-39. ACM Press, New York (2003)

3. Sunikka, A., Bragge, J.: Applying text-mining to personalization and customization research literature - Who, what and where? Expert Systems with Applications 39(11), 10049-10058 (2012)

4. Sung, J., Grinter, R.E., Christensen, H.I.: "Pimp My Roomba": designing for personalization. In: Proc. CHI 2009, pp. 193-196. ACM Press, New York (2009)

5. Ansari, A., Mela, C.F.: E-customization. J. of Marketing Research XL, 131-145 (May 2003)

6. Häkkila, J., Chatfield, C.: Personal customisation of mobile phones: a case study. In: Proc. NordiCHI 2006, pp. 409-412. ACM Press, New York (2006)

7. Ventä, L., Isomursu, M., Ahtinen, A., Ramiah, S.: My phone is part of my soul. In: Proc. UBICOMM 2008, pp. 311-317. IEEE Computer Society (2008)

8. Tossell, C.C., Kortum, P., Shepard, C., Rahmati, A., Zhong, L.: An empirical analysis of smartphone personalisation: measurement and user variability. Behaviour \& Information Technology, iFirst, 1-16 (2012)

9. Blom, J.O., Monk, A.F.: Theory of personalization of appearance: why users personalize their pcs and mobile phones. Hum.-Comput. Interact. 18(3), 193-228 (2003)

10. Page, S.R., Johnsgard, T.J., Albert, Allen, C.D.: User customization of a word processor. In: Proc. CHI 1996, pp. 340-346. ACM Press, New York (1996) 
11. Marathe, S., Sundar, S.S.: What drives customization?: control or identity? In: Proc. CHI 2011. ACM Press, New York (2011)

12. Blom, J.: Personalization-a taxonomy. In: Ext. Abstracts CHI 2000, pp. 313-314. ACM Press (2000)

13. Monk, A.F., Blom, J.O.: A theory of personalisation of appearance: quantitative evaluation of qualitatively derived data. Behavior and Information Technology 26(3), 237-246 (2007)

14. Suunto, http://www.suunto.fi/us/en/products/Outdoor_Sports _Instruments/suunto-ambit/suunto-ambit-black-hr

15. Deci, E.L., Ryan, R.M.: The "what" and "why" of goal pursuits: Human needs and the self-determination of behaviour. Psychological Inquiry 11, 227-268 (2000) 